PROCEEDINGS OF THE

AMERICAN MATHEMATICAL SOCIETY

Volume 126, Number 6, June 1998, Pages 1783-1790

S 0002-9939(98)04241-5

\title{
ANALYTIC SUBGROUPS OF THE REALS
}

\author{
MIKLÓS LACZKOVICH
}

(Communicated by Andreas R. Blass)

\begin{abstract}
We prove that every analytic proper subgroup of the reals can be covered by an $F_{\sigma}$ null set. We also construct a proper Borel subgroup $G$ of the reals that cannot be covered by countably many sets $A_{i}$ such that $A_{i}+A_{i}$ is nowhere dense for every $i$.
\end{abstract}

Let $G$ be an (additive) proper subgroup of the reals. It is well-known that if $G$ is measurable then $G$ is null, and if $G$ has the Baire property then $G$ is of the first category. This implies that if $G$ is analytic, then $G$ must be a first category null set. In the next theorem we prove a somewhat stronger statement.

Theorem 1. Every analytic proper subgroup of the reals can be covered by an $F_{\sigma}$ null set.

The proof of Theorem 1 is based on two results. The first is due to Gy. Petruska [3], and states that if an analytic set $H \subset \mathbf{R}$ cannot be covered by an $F_{\sigma}$ null set, then there is a closed set $F$ such that each portion of $F$ is of positive measure and $H$ is residual (comeager) in $F$. (By a portion of a set $F \subset \mathbf{R}$ we mean a nonempty and relative open subset of $F$.) If $A, B$ are arbitrary portions of $F$, then they are measurable sets of positive measure, and thus the set $A+B=\{x+y: x \in A, y \in B\}$ has nonempty interior. As we shall prove in the next lemma, this implies that whenever a set $H$ is residual in $F$, then $H+H$ is residual in an interval. In our case, however, $H$ is an analytic group, and hence $H+H=H$ is of first category. This contradiction proves the theorem.

Lemma 2. Let $F_{1}$ and $F_{2}$ be closed subsets of $\mathbf{R}$, and suppose that whenever $A_{i}$ is a portion of $F_{i}(i=1,2)$ then $\operatorname{int}\left(A_{1}+A_{2}\right) \neq \emptyset$. If $H_{i}$ is residual in $F_{i}(i=1,2)$, then $H_{1}+H_{2}$ is residual in any interval contained in $F_{1}+F_{2}$.

Proof. Let $I_{0}$ be an interval contained in $F_{1}+F_{2}$. We shall play the Banach-Mazur game in $I_{0}$ (as discribed in Chapter 6 of [2]), with the second player winning if the intersection of the intervals (moves) is a subset of $H_{1}+H_{2}$. We give a winning strategy for the second player; by Theorem 6.1 of [2], this will prove that $H_{1}+H_{2}$ is residual in $I_{0}$.

We may assume that $H_{i}$ is a dense $G_{\delta}$ subset of $F_{i}$. Let $H_{i}=\bigcap_{n=1}^{\infty} G_{i}^{n}(i=1,2)$, where $G_{i}^{n}$ is open (in $\mathbf{R}$ ) and dense in $F_{i}$ for every $i=1,2$ and $n=1,2, \ldots$ Suppose

Received by the editors February 20, 1996 and, in revised form, November 21, 1996.

1991 Mathematics Subject Classification. Primary 04A15.

This work was completed when the author had a visiting position at the Mathematical Institute of the Hungarian Academy of Sciences. Also supported by the Hungarian National Foundation for Scientific Research, Grant T016094. 
that the first move of the first player is the interval $I_{1}$. Since $H_{1}+H_{2}$ is dense in $F_{1}+F_{2}$, there are points $x_{1}^{1} \in H_{1}, x_{2}^{1} \in H_{2}$ such that $x_{1}^{1}+x_{2}^{1} \in \operatorname{int} I_{1}$. Then there are open intervals $J_{i}^{1}$ such that $x_{i}^{1} \in J_{i}^{1}, \operatorname{cl} J_{i}^{1} \subset G_{i}^{1}(i=1,2)$, and $J_{1}^{1}+J_{2}^{1} \subset I_{1}$. By assumption, the set

$$
U_{1}=\left(J_{1}^{1} \cap F_{1}\right)+\left(J_{2}^{1} \cap F_{2}\right)
$$

has nonempty interior. Let the first move of the second player be any closed interval $I_{2} \subset U_{1}$.

Let the second move of the first player be the interval $I_{3}$. Since $I_{3} \subset I_{2} \subset U_{1}$ and $H_{i}$ is dense in $F_{i}$, there are points $x_{i}^{2} \in H_{i} \cap J_{i}^{1}$ such that $x_{1}^{2}+x_{2}^{2} \in \operatorname{int} I_{3}$. Then there are open intervals $J_{i}^{2}$ such that $x_{i}^{2} \in J_{i}^{2}, \operatorname{cl} J_{i}^{2} \subset J_{i}^{1} \cap G_{i}^{2}(i=1,2)$, and $J_{1}^{2}+J_{2}^{2} \subset I_{3}$. By assumption, the set

$$
U_{2}=\left(J_{1}^{2} \cap F_{1}\right)+\left(J_{2}^{2} \cap F_{2}\right)
$$

has nonempty interior. Let the second move of the second player be any closed interval $I_{4} \subset U_{2}$, etc.

We have to show that if the second player sticks to this strategy, then $\bigcap_{n=1}^{\infty} I_{n} \subset$ $H_{1}+H_{2}$ holds. We have $I_{2 n} \subset J_{1}^{n}+J_{2}^{n}$ for every $n$, and cl $J_{i}^{n+1} \subset J_{i}^{n} \cap G_{i}^{n}$ for every $n$ and $i=1,2$. Let $x \in \bigcap_{n=1}^{\infty} I_{2 n}$, and let $x=x_{1}^{n}+x_{2}^{n}$, where $x_{i}^{n} \in J_{i}^{n}$. If $\left(x_{1}, x_{2}\right)$ is the limit of a convergent subsequence of $\left(x_{1}^{n}, x_{2}^{n}\right)$, then $x_{i} \in \bigcap_{n=1}^{\infty} \operatorname{cl} J_{i}^{n} \subset H_{i} \quad(i=1,2)$ and $x=x_{1}+x_{2} \in H_{1}+H_{2}$.

We remark that the statement of Theorem 1 is true in every locally compact and second countable group with the Haar measure. Indeed, such a group is (homeomorphic to) a Polish space. Since Petruska's theorem is valid for every Polish space (with any continuous Borel measure on it), it is easy to check that the proof of Theorem 1 works also in this more general setting.

The statement of Lemma 2 does not remain valid if we replace the condition on the sets $F_{1}, F_{2}$ by $\operatorname{int}\left(F_{1}+F_{2}\right) \neq \emptyset$. Consider the following example. Let $H \subset[0,1]$ be a set containing exactly one point of each interval contiguous to the Cantor ternary set $C$. Let $F=C \cup H$. Then $F$ is closed, and $F+F=C+C=[0,2]$. The set $H$ is a residual (moreover, dense open) subset of $F$, but $H+H$, being countable, is not residual in any interval.

Petruska's theorem was generalized by S. Solecki as follows. Let $\mathcal{F}$ be an arbitrary system of closed subsets of $\mathbf{R}$, and let $\mathcal{F}_{\omega}$ denote the family of all sets that can be covered by countably many elements of $\mathcal{F}$. Solecki proved in $[4$, Theorem 1] that if $H$ is an analytic set with $H \notin \mathcal{F}_{\omega}$, then $H$ contains a $G_{\delta}$ set $U$ such that $U \notin \mathcal{F}_{\omega}$. Let $\mathcal{F}=\{F \subset \mathbf{R}: F$ is closed, and each portion of $F$ contains two portions, $A_{1}$ and $A_{2}$, such that $A_{1}+A_{2}$ is nowhere dense $\}$.

If we combine Solecki's theorem with Lemma 2, then we obtain the following statement: every proper analytic subgroup of $\mathbf{R}$ can be covered by countable many closed sets belonging to $\mathcal{F}$.

This result motivates the following question: is it true that every proper analytic subgroup of $\mathbf{R}$ can be covered by countably many closed sets, $F_{1}, F_{2}, \ldots$ such that $F_{n}+F_{n}$ is nowhere dense for every $n$ ? Our next aim is to show that the answer is negative, even for Borel subgroups.

Theorem 3. There exists a proper Borel subgroup $G \subset \mathbf{R}$ that cannot be covered by countably many sets $A_{i}$ such that $A_{i}+A_{i}$ is nowhere dense for every $i$. Consequently, if an $F_{\sigma}$ set $E$ covers $G$ then $\operatorname{int}(E+E) \neq \emptyset$. 
We shall prove this through the following result.

Theorem 4. There exists a $G_{\delta}$ set $A \subset \mathbf{R}$ such that the elements of $A$ are linearly independent over the rational numbers, and $A$ cannot be covered by countably many sets $A_{i}$ such that $A_{i}+A_{i}$ is nowhere dense for every $i$.

First we infer Theorem 3 from Theorem 4 . Let $A$ be the set given by Theorem 4 , and let $G$ be the group generated by $A$. It is enough to show that $G$ is Borel and $G \neq \mathbf{R}$. Let $k \in \mathbf{N}$ be fixed, and put

$$
A_{k}=\left\{\left(x_{1}, \ldots, x_{k}\right): x_{1}, \ldots, x_{k} \text { are distinct elements of } A\right\} .
$$

It is easy to see that $A_{k}$ is a $G_{\delta}$ subset of $\mathbf{R}^{k}$. Let $n_{1}, \ldots, n_{k}$ be fixed nonzero integers, and let $f: A_{k} \rightarrow \mathbf{R}$ be defined by

$$
f\left(x_{1}, \ldots, x_{k}\right)=n_{1} x_{1}+\ldots+n_{k} x_{k} \quad\left(\left(x_{1}, \ldots, x_{k}\right) \in A_{k}\right) .
$$

Then $f$ is continuous and, as the elements of $A$ are linearly independent over $\mathbf{Q}$, $f^{-1}(y)$ is finite for every $y \in f\left(A_{k}\right)$. This implies that the set $G_{n_{1} n_{2} \ldots n_{k}}=f\left(A_{k}\right)$ is Borel (see [1, Corollary 5, p. 498]). Since

$$
G=\{0\} \cup \bigcup\left\{G_{n_{1} n_{2} \ldots n_{k}}: n_{1}, \ldots, n_{k} \in \mathbf{Z} \backslash\{0\}\right\},
$$

it follows that $G$ is Borel.

Each $G_{n_{1} n_{2} \ldots n_{k}}$ is a null set, since otherwise $G_{n_{1} n_{2} \ldots n_{k}}+G_{n_{1} n_{2} \ldots n_{k}}$ would contain an interval, contradicting the condition that the elements of $A$ are linearly independent over $\mathbf{Q}$. This proves that $G$ is also null, and thus $G \neq \mathbf{R}$.

We remark that $A+A$, being a subset of $G$, is of first category. Thus we obtain the following corollary.

Corollary 5. There exists a $G_{\delta}$ set $A \subset \mathbf{R}$ such that $A+A$ is of first category, but $A$ cannot be covered by countably many sets $A_{i}$ such that $A_{i}+A_{i}$ is nowhere dense for every $i$.

We shall use the following notation. For every compact set $K \subset \mathbf{R}$ we shall denote by $\bar{K}$ the convex hull of $K$; that is, the interval $[\min K, \max K]$. Clearly, the components of $\bar{K} \backslash K$ are the bounded intervals contiguous to $K$. Let $\mathcal{K}$ be a system of nowhere dense perfect subsets of $\mathbf{R}$. We shall say that $\mathcal{K}$ is regular, if the elements of $\mathcal{K}$ are pairwise disjoint, and for every $K \in \mathcal{K}$ and for every component $I$ of $\bar{K} \backslash K$ there is $L \in \mathcal{K}$ with $L \subset I$.

Lemma 6. Let $\mathcal{K}$ be a countable and regular system of nowhere dense perfect subsets of $\mathbf{R}$, and put $A=\operatorname{cl}(\bigcup \mathcal{K}) \backslash(\bigcup \mathcal{K})$. If $A=\bigcup_{i=1}^{\infty} A_{i}$, then there is an index $i$ and a set $K \in \mathcal{K}$ such that $K \subset \operatorname{cl} A_{i}$.

Proof. Let $K_{1}, K_{2}, \ldots$ be an enumeration of the elements of $\mathcal{K}$. Suppose that the statement of the lemma is false. Then $K_{1} \not \subset \mathrm{cl} A_{1}$, and we may choose a point $x_{1} \in K_{1} \backslash \operatorname{cl} A_{1}$. Since $K_{1}$ is perfect, there is a component $I_{1}=\left(a_{1}, b_{1}\right)$ of $\overline{K_{1}} \backslash K_{1}$ for which $I_{1} \subset\left(x_{1}-1, x_{1}+1\right)$, and $\operatorname{cl} I_{1} \cap A_{1}=\emptyset$. By the regularity of $\mathcal{K}$ we can choose an index $n_{1}$ such that $K_{n_{1}} \subset I_{1}$. Then $K_{n_{1}} \not \subset \mathrm{cl} A_{2}$ (since otherwise the statement of the lemma would be true) and we can choose a point $x_{2} \in K_{n_{1}} \backslash \mathrm{cl} A_{2}$. Since $K_{n_{1}}$ is nowhere dense and perfect, and the elements of $\mathcal{K}$ are pairwise disjoint, there is a component $I_{2}=\left(a_{2}, b_{2}\right)$ of $\overline{K_{n_{1}}} \backslash K_{n_{1}}$ such that $I_{2} \subset\left(x_{2}-(1 / 2), x_{2}+(1 / 2)\right)$, 
$\operatorname{cl} I_{2} \cap A_{2}=\emptyset$, and

$$
\left(\operatorname{cl} I_{2}\right) \cap \bigcup_{i=1}^{n_{1}-1} K_{i}=\emptyset .
$$

Then we choose an element $K_{n_{2}} \in \mathcal{K}$ with $K_{n_{2}} \subset I_{2}$ and a component $I_{3}=\left(a_{3}, b_{3}\right)$ of $\overline{K_{n_{2}}} \backslash K_{n_{2}}$ such that $\left|I_{3}\right| \leq 2 / 3$ and $\mathrm{cl} I_{3}$ is disjoint from the sets $A_{3}$ and $\bigcup_{i=1}^{n_{2}-1} K_{i}$. Repeating this process, we can define the sets $K_{n_{j}}$ and the intervals $I_{j}=\left(a_{j}, b_{j}\right)$ for every $j=1,2, \ldots$ Let $\bigcap_{j=1}^{\infty} \operatorname{cl} I_{j}=\{x\}$. Then $x \in \operatorname{cl}(\bigcup \mathcal{K})$, since $a_{j} \in K_{n_{j-1}} \subset \bigcup \mathcal{K}$ and $a_{j} \rightarrow x$. On the other hand, $x \notin \bigcup \mathcal{K}$, since $\operatorname{cl} I_{j} \cap \bigcup_{i=1}^{n_{j-1}-1} K_{i}=\emptyset$ for every $j$. Therefore $x \in \operatorname{cl}(\bigcup \mathcal{K}) \backslash(\bigcup \mathcal{K})=A$, and hence $x \in A_{i}$ for some $i$. However, $\operatorname{cl} I_{i} \cap$ $\operatorname{cl} A_{i}=\emptyset$ implies $x \notin A_{i}$, a contradiction.

Lemma 7. Let $\mathcal{K}$ be a countable and regular system of nowhere dense perfect subsets of $\mathbf{R}$, and suppose that $\operatorname{int}(K+K) \neq \emptyset$ for every $K \in \mathcal{K}$. Let

$$
A=\operatorname{cl}(\bigcup \mathcal{K}) \backslash(\bigcup \mathcal{K}) \text {. }
$$

If $A=\bigcup_{i=1}^{\infty} A_{i}$, then there is an index $i$ such that $A_{i}+A_{i}$ is dense in an interval.

Proof. Since

$$
\operatorname{cl}\left(A_{i}+A_{i}\right) \supset\left(\operatorname{cl} A_{i}\right)+\left(\operatorname{cl} A_{i}\right)
$$

for every $i$, the statement is immediate from the previous lemma.

In the sequel we shall construct a system $\mathcal{K}$ satisfying the conditions of Lemma 7 such that the elements of the set $A$ defined by (1) are indepentent over the rationals. Since $A$ is $G_{\delta}$, this will prove Theorem 4 .

We shall say that a set $H \subset \mathbf{R}$ is a figure, if $H$ is the union of finitely many closed segments.

Lemma 8. Let $I$ and $J$ be closed intervals, and let $F \subset \mathbf{R}$ be a nowhere dense closed set not containing the endpoints of $I$ and $J$. Then there are figures $A \subset I \backslash F$ and $B \subset J \backslash F$ such that $A+B=I+J$.

Proof. Let $I=[a, b]$ and $J=[c, d]$. First we show that for every $x \in I+J$ there are numbers $x_{1} \in I \backslash F, x_{2} \in J \backslash F$ with $x=x_{1}+x_{2}$. This is clear if $x=a+c$ or $x=b+d$, since $a, b, c, d \notin F$. If $x \in I+J$ is not of this form, then $a+c<x<b+d$. In this case $(x-b, x-a) \cap(c, d)$ is a nonempty open interval, and thus the set

$$
D=[(x-b, x-a) \cap(c, d)] \backslash[((-F)+x) \cup F]
$$

is nonempty. If $x_{2} \in D$ then $x_{2} \in(c, d) \backslash F \subset J \backslash F$ and $x_{1} \stackrel{\text { def }}{=} x-x_{2} \in(a, b) \backslash F \subset I \backslash F$. Let

$$
A_{k}=\{x \in I: \operatorname{dist}(x, F)>1 / k\}, \quad B_{k}=\{x \in J: \operatorname{dist}(x, F)>1 / k\} .
$$

By the preceding argument we have

$$
I+J=\bigcup_{k=1}^{\infty}\left(A_{k}+B_{k}\right) .
$$

Since the sets $A_{k}+B_{k}$ are relatively open in the compact interval $I+J$, this implies that $A_{k}+B_{k}=I+J$ for some $k$. Fix a $k$ with this property, and put

$$
A=\{x \in I: \operatorname{dist}(x, F) \geq 1 / k\}, \quad B=\{x \in J: \operatorname{dist}(x, F) \geq 1 / k\} .
$$

Then $A+B=I+J$, and it is easy to see that $A$ and $B$ are figures. 
Lemma 9. Suppose that $H \subset \mathbf{R}$ is a figure and $F \subset \operatorname{int} H$ is nowhere dense and closed. Then there is a figure $H^{\prime} \subset H \backslash F$ such that $H^{\prime}+H^{\prime}=H+H$.

Proof. Let $H=\bigcup_{i=1}^{n}\left[a_{i}, b_{i}\right]$. By Lemma 8 , for every $1 \leq i, j \leq n$ there are figures $A_{i, j} \subset\left[a_{i}, b_{i}\right] \backslash F$ and $B_{i, j} \subset\left[a_{j}, b_{j}\right] \backslash F$ such that

$$
A_{i, j}+B_{i, j}=\left[a_{i}, b_{i}\right]+\left[a_{j}, b_{j}\right] .
$$

Let

$$
H^{\prime}=\bigcup_{i=1}^{\infty} \bigcup_{j=1}^{\infty}\left(A_{i, j} \cup B_{i, j}\right) .
$$

Then $H^{\prime}$ is a figure, $H^{\prime} \subset H \backslash F$, and $H^{\prime}+H^{\prime}=H+H$.

Lemma 10. If $A \subset(a, b)$ is a set of first category, then there is a nowhere dense perfect set $K \subset[a, b] \backslash A$ such that $K+K=[2 a, 2 b]$.

Proof. We may assume that $A$ is dense in $(a, b)$, and $A=\bigcup_{n=1}^{\infty} F_{n}$, where $F_{n} \subset$ $(a, b)$ is nowhere dense and closed for every $n$. We define a sequence of figures $H_{n}$ as follows. Put $F_{0}=\emptyset$ and $H_{0}=[a, b]$. Let $n \geq 0$ and suppose that the figure $H_{n}$ has been defined in such a way that the endpoints of the components of $H_{n}$ do not belong to $A$. Applying the previous lemma, we can find a figure $B \subset H_{n} \backslash F_{n}$ such that $B+B=H_{n}+H_{n}$. By enlarging the components of $B$, we may assume that the endpoints of the components of $B$ do not belong to $A$. Also, by adding new intervals to $B$ if necessary, we can suppose that each component of $H_{n}$ contains at least two components of $B$. Then we put $H_{n+1}=B$. In this way we define the figures $H_{n}$ for every $n$ so that $H_{n+1} \subset H_{n} \backslash F_{n}(n=1,2, \ldots)$, and

$$
[2 a, 2 b]=H_{0}+H_{0}=H_{1}+H_{1}=\ldots .
$$

Let $K=\bigcap_{n=1}^{\infty} H_{n}$. It is easy to see, using the fact that each $H_{n}$ is compact, that $K+K=[2 a, 2 b]$. Since $A$ is dense in $[a, b]$ and $K \cap A=\emptyset$, it follows that $K$ is nowhere dense. Finally, the condition that each component of $H_{n}$ contains at least two components of $H_{n+1}$ implies that $K$ is perfect.

Let $\mathcal{A}$ be a system of subsets of $\mathbf{R}$. Suppose that, whenever $A_{1}, \ldots, A_{n}$ are distinct elements of $\mathcal{A}$, and $r_{1}, \ldots, r_{n}$ are nonzero rational numbers, then

$$
0 \notin r_{1} A_{1}+\ldots+r_{n} A_{n} .
$$

In this case we shall say that the system $\mathcal{A}$ is linearly independent over $\mathbf{Q}$.

Lemma 11. Let $K_{1}, K_{2}, \ldots$ be nonempty compact sets such that the system $\left\{K_{1}, K_{2}, \ldots\right\}$ is linearly independent over $\mathbf{Q}$. Then for every sequence of intervals $\left[a_{i}, b_{i}\right](i=1,2, \ldots)$ there are nowhere dense perfect sets $P_{i} \subset\left[a_{i}, b_{i}\right](i=1,2, \ldots)$ such that $\operatorname{int}\left(P_{i}+P_{i}\right) \neq \emptyset$ for every $i$, and the system $\left\{K_{1}, K_{2}, \ldots, P_{1}, P_{2}, \ldots\right\}$ is also linearly independent over $\mathbf{Q}$.

Proof. Clearly, $0 \notin K_{i}$ for every $i$. Also, if $i_{1}, \ldots, i_{n}$ are distinct indices and $r_{1}, \ldots, r_{n} \in \mathbf{Q} \backslash\{0\}$, then the set $F=r_{1} K_{i_{1}}+\ldots+r_{n} K_{i_{n}}$ is nowhere dense. Indeed, $F$ is compact, and thus, if $F$ is not nowhere dense, then its interior is nonempty. In this case we can choose an index $i_{n+1}$ distinct from $i_{1}, \ldots, i_{n}$, and a nonzero rational number $r_{n+1}$ such that

$$
0 \in r_{1} K_{i_{1}}+\ldots+r_{n} K_{i_{n}}+r_{n+1} K_{i_{n+1}},
$$


since $K_{i_{n+1}}$ contains a nonzero element. This, however, contradicts the condition that the system $\left\{K_{1}, K_{2}, \ldots\right\}$ is linearly independent over $\mathbf{Q}$, and thus $F$ must be nowhere dense.

Let $A$ denote the union of all sets of the form $r_{1} K_{i_{1}}+\ldots+r_{n} K_{i_{n}}$, where $r_{1}, \ldots, r_{n} \in \mathbf{Q} \backslash\{0\}$ and $i_{1}, \ldots, i_{n}$ are distinct indices. Then $A$ is of the first category. Let $c, d \in\left[a_{1}, b_{1}\right] \backslash A, c<d$. By the previous lemma, there is a nowhere dense perfect set $P_{1} \subset[c, d] \backslash A$ such that $P_{1}+P_{1}=[2 c, 2 d]$. It is easy to see, using $P_{1} \cap A=\emptyset$, that the system $\left\{K_{1}, K_{2}, \ldots, P_{1}\right\}$ is linearly independent over $\mathbf{Q}$. Repeating this argument, we find a nowhere dense perfect set $P_{2} \subset\left[a_{2}, b_{2}\right]$ such that $\operatorname{int}\left(P_{2}+P_{2}\right) \neq \emptyset$, and the system $\left\{K_{1}, K_{2}, \ldots, P_{1}, P_{2}\right\}$ is linearly independent over Q. Continuing this process, we find the sets $P_{i}$ with the required properties.

Now we turn to the proof of Theorem 4 . We shall construct a system $\mathcal{K}$ with the properties described in Lemma 7 such that the elements of the set $A$ defined by (1) are linearly independent over the rational numbers. Let $\Sigma$ denote the set of those finite sequences

$$
\left(n_{0}, n_{1}, \ldots, n_{j}, r_{1}, \ldots, r_{j}\right)
$$

in which $n_{0}, \ldots, n_{j} \in \mathbf{N}, n_{1}, \ldots, n_{j}$ are distinct, and $r_{1}, \ldots, r_{j} \in \mathbf{Q} \backslash\{0\}$. Let $\sigma_{1}, \sigma_{2}, \ldots$ be an enumeration of the elements of $\Sigma$. By Lemma 11, we can find nowhere dense perfect sets $K_{n}^{1} \subset[0,1](n=1,2, \ldots)$ such that $\operatorname{int}\left(K_{n}^{1}+K_{n}^{1}\right) \neq \emptyset$ for every $n$, the system $\left\{K_{1}^{1}, K_{2}^{1}, \ldots\right\}$ is linearly independent over $\mathbf{Q}$, and the convex hulls $\overline{K_{n}^{1}}$ are pairwise disjoint. We also put $S_{1}=\emptyset$.

Let $k \geq 1$, and suppose that we have defined the finite set $S_{k} \subset \Sigma$ and the nowhere dense perfect sets $K_{n}^{i}(i=1, \ldots, k, n=1,2, \ldots)$ with the following properties:

(i) $\operatorname{int}\left(K_{n}^{i}+K_{n}^{i}\right) \neq \emptyset$ for every $i \leq k$ and $n=1,2, \ldots$;

(ii) the system $\mathcal{K}_{k}=\left\{K_{n}^{i}: i=1, \ldots, k, n=1,2, \ldots\right\}$ is linearly independent over $\mathbf{Q}$

(iii) for every $i \leq k$, the intervals $\overline{K_{n}^{i}}(n=1,2, \ldots)$ are pairwise disjoint and are shorter than $1 / i$;

(iv) whenever $i<k, n \in \mathbf{N}$ and $I$ is a component of $\overline{K_{n}^{i}} \backslash K_{n}^{i}$, there is exactly one index $m$ such that $K_{m}^{i+1} \subset I$;

(v) for every $i<k$ and $n \in \mathbf{N}$, there is $m \in \mathbf{N}$ such that $K_{n}^{i+1}$ is contained in one of the components of $\overline{K_{m}^{i}} \backslash K_{m}^{i}$; finally,

(vi) if $\left(n_{0}, n_{1}, \ldots, n_{j}, r_{1}, \ldots, r_{j}\right) \in S_{k}$, then $n_{0}<k$ and

$$
0 \notin r_{1} F_{n_{1}}^{n_{0}, k}+\ldots+r_{j} F_{n_{j}}^{n_{0}, k}
$$

where

$$
F_{m}^{n_{0}, k}=\bigcup\left\{\overline{K_{n}^{k}}: \overline{K_{n}^{k}} \subset \overline{K_{m}^{n_{0}}}\right\} \quad(m \in \mathbf{N}) .
$$

Let $I_{1}, I_{2}, \ldots$ be an enumeration of those intervals that are components of any of the sets $\overline{K_{n}^{k}} \backslash K_{n}^{k}(n=1,2, \ldots)$. By Lemma 11, there are nonempty sets $P_{j} \subset I_{j}$ such that the system $\mathcal{K}_{k} \cup\left\{P_{1}, P_{2}, \ldots\right\}$ is linearly independent over $\mathbf{Q}$. Let $y_{j} \in P_{j}$ for every $j$. Let $\sigma_{p}=\left(n_{0}, n_{1}, \ldots, n_{j}, r_{1}, \ldots, r_{j}\right)$ be the first element of the sequence $\sigma_{1}, \sigma_{2}, \ldots$ for which $n_{0} \leq k$ and $\sigma_{p} \notin S_{k}$. Then we define $S_{k+1}=S_{k} \cup\left\{\sigma_{p}\right\}$. Let

$$
H_{m}^{n_{0}}=\bigcup\left\{K_{n}^{i}: n_{0} \leq i \leq k, \overline{K_{n}^{i}} \subset \overline{K_{m}^{n_{0}}}\right\} \cup\left\{y_{j}: y_{j} \in \overline{K_{m}^{n_{0}}}\right\} .
$$


It is easy to check, using (iv) and (v), that $H_{m}^{n_{0}}$ is compact for every $m$. This implies that the set

$$
H=r_{1} H_{n_{1}}^{n_{0}}+\ldots+r_{j} H_{n_{j}}^{n_{0}}
$$

is also compact. Since $\mathcal{K}_{k} \cup\left\{P_{1}, P_{2}, \ldots\right\}$ is linearly independent over $\mathbf{Q}$, it follows that $0 \notin H$, and thus there is $\delta>0$ such that $H \cap(-\delta, \delta)=\emptyset$. Let

$$
\eta=\min \left(\frac{1}{k+1}, \frac{\delta}{\left|r_{1}\right|+\ldots+\left|r_{j}\right|}\right) .
$$

Applying Lemma 11, we obtain nowhere dense perfect sets $K_{n}^{k+1} \subset\left(y_{n}, y_{n}+\eta\right) \cap I_{n}$ such that $\operatorname{int}\left(K_{n}^{k+1}+K_{n}^{k+1}\right) \neq \emptyset$ for every $n$, and the system

$$
\mathcal{K}_{k+1}=\left\{K_{n}^{i}: i=1, \ldots, k+1, n=1,2, \ldots\right\}
$$

is linearly independent over $\mathbf{Q}$. Then

$$
0 \notin r_{1} F_{n_{1}}^{n_{0}, k+1}+\ldots+r_{j} F_{n_{j}}^{n_{0}, k+1}
$$

follows from the choice of $\eta$. Since $F_{m}^{i, k+1} \subset F_{m}^{i, k}$ for every $i<k$ and $m \in \mathbf{N}$, (vi) remains valid for $k+1$. It is clear from the construction that (i)-(v) are also satisfied by $\mathcal{K}_{k+1}$.

In this way we have defined, by induction, the sets $S_{k}$ and $K_{n}^{k}$ for every $k, n \in \mathbf{N}$. Clearly, $\mathcal{K}=\left\{K_{n}^{i}: i, n \in \mathbf{N}\right\}$ is a countable and regular system of nowhere dense perfect sets such that int $(K+K) \neq \emptyset$ for every $K \in \mathcal{K}$. In order to complete the proof, we have to show that the elements of the set $A$ defined by (1) are linearly independent over the rationals. First we remark that the set

$$
M^{k}=\bigcup_{n=1}^{\infty} \overline{K_{n}^{k}}
$$

contains $A$ for every $k=1,2, \ldots$ Indeed, the set

$$
L^{k}=M^{k} \cup \bigcup_{i=1}^{k-1} \bigcup_{n=1}^{\infty} K_{n}^{i}
$$

is closed and contains $\bigcup \mathcal{K}$. Thus we have $\operatorname{cl}(\bigcup \mathcal{K}) \subset L^{k}$ and

$$
A=\operatorname{cl}(\bigcup \mathcal{K}) \backslash \bigcup \mathcal{K} \subset L^{k} \backslash \bigcup \mathcal{K} \subset M^{k} .
$$

Let $x_{1}, \ldots, x_{j}$ be distinct elements of $A$ and let $r_{1}, \ldots, r_{j} \in \mathbf{Q} \backslash\{0\}$. We show that $r_{1} x_{1}+\ldots+r_{j} x_{j} \neq 0$. Choose a positive integer $n_{0}$ with

$$
1 / n_{0}<\min \left\{\left|x_{i_{2}}-x_{i_{1}}\right|: 1 \leq i_{1}<i_{2} \leq j\right\}
$$

Since $A \subset M^{n_{0}}$, there are indices $n_{1}, \ldots, n_{j}$ such that $x_{i} \in \overline{K_{n_{i}}^{n_{0}}}(i=1, \ldots, j)$. By the choice of $n_{0}$ and by (iii), the indices $n_{1}, \ldots, n_{j}$ are distinct. Let

$$
\left(n_{0}, n_{1}, \ldots, n_{j}, r_{1}, \ldots, r_{j}\right)=\sigma_{p} .
$$

We put $k=n_{0}+p$; then it follows from the construction that $\sigma_{p} \in S_{k}$. We have

$$
x_{i} \in \overline{K_{n_{i}}^{n_{0}}} \cap A \subset \overline{K_{n_{i}}^{n_{0}}} \cap M^{k}=F_{n_{i}}^{n_{0}, k}
$$

for every $i=1, \ldots, j$. Therefore, by (vi) we obtain $r_{1} x_{1}+\ldots+r_{j} x_{j} \neq 0$, which completes the proof. 


\section{REFERENCES}

[1] K. Kuratowski, Topology, Vol. I. Academic Press, 1966. MR 36:840

[2] J. C. Oxtoby, Measure and Category. Springer, 1971. MR 52:14213

[3] Gy. Petruska, On Borel sets with small cover, Real Analysis Exchange 18 (1992-93) (2), 330-338. MR 95g:28003a

[4] S. Solecki, Covering analytic sets by families of closed sets, Journal of Symbolic Logic 59 (1994), 1022-1031. MR 95g:54033

Department of Analysis, Eötvös Loránd University, Budapest, Muzeum krt. 6-8, HUNGARY 1088

E-mail address: laczk@cs.elte.hu 